\title{
CONTRE-PROPAGANDE SUR L'INDOCHINE. LA FRANCE OFFICIELLE FACE À L'ANTICOLONIALISME EN BELGIQUE FRANCOPHONE (1946-1950)
}

\section{Catherine Lanneau}

\section{Presses Universitaires de France | « Guerres mondiales et conflits contemporains »}

2011/1 n $241 \mid$ pages 75 à 90

ISSN 0984-2292

ISBN 9782130587170

Article disponible en ligne à l'adresse :

http://www.cairn.info/revue-guerres-mondiales-et-conflits-contemporains-2011-1-page-75.htm

\section{!Pour citer cet article :}

Catherine Lanneau, «Contre-propagande sur l'Indochine. La France officielle face à l'anticolonialisme en Belgique francophone (1946-1950) », Guerres mondiales et conflits contemporains 2011/1 ( $\mathrm{n}^{\circ} 241$ ), p. 75-90.

DOI 10.3917/gmcc.241.0075

Distribution électronique Cairn.info pour Presses Universitaires de France.

(C) Presses Universitaires de France. Tous droits réservés pour tous pays.

La reproduction ou représentation de cet article, notamment par photocopie, n'est autorisée que dans les limites des conditions générales d'utilisation du site ou, le cas échéant, des conditions générales de la licence souscrite par votre établissement. Toute autre reproduction ou représentation, en tout ou partie, sous quelque forme et de quelque manière que ce soit, est interdite sauf accord préalable et écrit de l'éditeur, en dehors des cas prévus par la législation en vigueur en France. Il est précisé que son stockage dans une base de données est également interdit. 


\section{CONTRE-PROPAGANDE SUR L'INDOCHINE. LA FRANCE OFFICIELLE FACE À L'ANTICOLONIALISME EN BELGIQUE FRANCOPHONE (1946-1950) ${ }^{1}$}

Pour de multiples raisons, liées notamment à la perte quasi concomitante du Congo et à la proximité géographique qui fit de la Belgique une base arrière pour le FLN, le MNA puis l'OAS, la guerre d'Algérie a suscité, dans l'opinion belge, une avalanche de réactions. Elle a également poussé certains à l'action, dans l'un ou l'autre sens. Par conséquent, elle a conduit la France à combattre ces divers réseaux d'aide et de soutien, en coopération avec les services belges. Mais, Paris a aussi tenté de contrôler le flot d'informations qui déferlait sur le pays voisin en réagissant systématiquement aux nouvelles jugées fausses et en s'employant à influencer les médias locaux avec plus ou moins de talent et de réussite ${ }^{2}$.

Si cet épisode est bien connu, on ne peut en dire autant de la réception en Belgique de l'autre grande guerre française de décolonisation. Cette guerre d'Indochine fut, elle aussi, un conflit de longue haleine, mais elle mobilisa nettement moins l'opinion française et, partant, l'opinion belge. Pour autant, il serait faux de penser que celle-ci n'y ait pas réagi et, qu'en retour, la France officielle - le Quai d'Orsay (Direction politique, Service de presse, direction générale des Relations culturelles), l'ambassade à Bruxelles et son attaché d'information - n'ait pas tenté d'influer sur la perception belge de cette guerre, et particulièrement sur celle des Belges de langue française.

Cet exposé se concentrera sur la première phase du conflit indochinois, du déclenchement des hostilités, fin 1946, au tournant que représente

1. Catherine Lanneau (1977) est docteur en histoire, titulaire d'un DEA en relations internationales et intégration européenne. Chargée de cours à l'université de Liège depuis octobre 2009, elle consacre ses principales recherches à l'histoire de Belgique, de Wallonie et des relations francobelges au $\mathrm{xx}^{\mathrm{e}}$ siècle et, en particulier, à l'action dans les médias de groupes de pression ou de réflexion. Elle a publié L'Inconnue française. La France et les Belges francophones 1944-1945 (PIEPeter Lang, 2008).

2. Voir surtout: D. Masset, Une affaire intérieure française? La Belgique et la guerre d'Algérie (1954-1956), Louvain-la-Neuve, Ciaco, 1988 ; J.-L. Doneux, et H. Le Paige, Le Front du Nord. Des Belges dans la guerre d'Algérie (1954-1962) [Pol-His], Bruxelles, De Boeck-RTBF, 1992. 
l'année $1950^{3}$. C'est à ce moment, en effet, qu'une guerre perçue comme coloniale se mue réellement en conflit de guerre froide, avec une implication croissante de la Chine communiste et une réévaluation de la situation par les États-Unis. 1950 est l'année de la guerre de Corée, de la radicalisation du Vietminh, de la reconnaissance de Bao Daï comme empereur du Vietnam par les États-Unis et la Grande-Bretagne et, à l'automne, de l'évacuation française catastrophique depuis Cao Bang et Langson dans le Haut-Tonkin. Nous envisagerons d'abord les réactions de l'opinion belge francophone face au conflit indochinois avant de nous concentrer sur l'impact des courants anticolonialistes et sur les réactions de la France à leur égard.

\section{L'OPINION BELGE FRANCOPHONE FACE À L'INDOCHINE}

Au lendemain de l'insurrection de Hanoï, survenue dans la nuit du 19 au 20 décembre 1946, la condamnation du Vietminh est virulente, de la droite catholique jusqu'à certains organes de gauche en passant par les libéraux. L'hebdomadaire libéral bruxellois Pourquoi Pas? fustige la " félonie " et la "cruauté asiatique " ${ }^{4}$ tandis que Le Soir (centre-gauche) comme la feuille socialiste liégeoise $\mathrm{La}$ Wallonie parlent d' "agression sournoise ${ }^{5}$. La gauche et surtout l'extrême gauche communiste sont cependant embarrassées, prises en tenaille entre leur anticolonialisme foncier et leur soutien au Gouvernement français. La tentation est forte de supposer une provocation colonialiste pour pousser Hô Chi Minh à la faute ${ }^{6}$ ou de renvoyer dos à dos Français et Vietnamiens ${ }^{7}$. Très vite pourtant, l'opposition à la politique de Paris et à une guerre présentée comme coloniale deviendra la norme chez les communistes belges, fidèles suiveurs du PCF, surtout à partir du printemps $1947^{\circ}$. Le monde socialiste ou socialisant demeurera, quant à lui, plus nuancé, la personnalité et la liberté d'appréciation des reporters, des rédacteurs ou des collaborateurs occasionnels jouant un rôle non négligeable. Le moniteur du Parti, Le Peuple, ouvrira ses colonnes à des socialistes français de toutes tendances, mais s'emploiera surtout à traiter de l'Indochine de manière neutre, voire détachée, le plus souvent par l'intermédiaire de dépêches AFP. La ligne anticolonialiste doit plutôt être cherchée dans des journaux de province classés à gauche mais sans lien avec le PSB comme Indépendance, quotidien de Charleroi proche des milieux de la Résistance.

3. Sur ce tournant et l'internationalisation qui en découle, voir F. Joyaux, La Nouvelle Question d'Extrême-Orient. T.I : l'ère de la guerre froide, Paris, Payot, 1985. La revue Guerres mondiales et conflits contemporains a en outre consacré son no 148 d'octobre 1987 à "L'Indochine de 1945 à 1952 ".

4. Pourquoi Pas?, 10/1/1947, p. 77.

5. Soir, 22/12/1946, p. 1 - Wallonie, 23/12/1946, p. 1.

6. Drapeau Ronge, 26/12/1946, p. 3.

7. Peuple, $27 / 12 / 1946$, p. 3.

8. C'est à ce moment en que communistes belges et français quittent leurs gouvernements respectifs. Sur l'attitude du PCF et, partant, du PCB face à la guerre d'Indochine, voir la publication de la thèse de A. Ruscio, Les Communistes français et la guerre d'Indochine, Paris, L'Harmattan, 1985. 
Durant l'année 1947, alors que les libéraux restent sur une ligne profrançaise, les catholiques - chrétiens de gauche et, dans une moindre mesure, conservateurs - ont davantage tendance à rechercher les causes profondes du combat nationaliste vietnamien et à les trouver dans les faiblesses et les erreurs de la France. Cette dernière n'ayant plus les moyens d'une politique de présence, la raison ne lui dicterait-elle pas de sauver à tout le moins son influence culturelle ? Derrière cette réflexion se cache bien sûr la défense des intérêts chrétiens en Extrême-Orient. En Indochine, près de dix pour cent de la population peut, en effet, être considérée comme catholique 9 . Avec la bénédiction de l'Église, qui pèse sur la rédaction de divers organes de presse ${ }^{10}$, les feuilles catholiques s'ouvrent à des sources provietnamiennes. La Gazette de Liège privilégie les dépêches américaines AP à celles de l'AFr, jugées partisanes, et accorde, on le verra, une large audience à des Belges, religieux ou non, revenus d'Indochine. Quant à Vers l'Avenir et à La Revue Nouvelle, ils ouvrent largement leurs pages au R. P. Camille Lozet qui revient d'une décennie passée au Laos et en Annam. Le missionnaire accuse ouvertement la France laïque d'avoir fait exploser, en cinquante ans de présence, la structure familiale annamite ${ }^{11}$. Début novembre 1947, Vers l'Avenir publiera une interview de l'abbé Huong, prêtre indochinois, tout aussi critique envers la France ${ }^{12}$.

On retrouve clairement, dans les thèses défendues sur l'Indochine par ces chrétiens belges, une proximité avec leurs homologues français, qu'ils soient liés à la revue Esprit ou à l'hebdomadaire Témoignage Chrétien ${ }^{13}$. La circulation des idées est réelle, préparant les échanges plus intenses encore qui auront lieu durant la guerre d'Algérie. En réalité, beaucoup de catholiques français et belges perçoivent les nationalistes vietnamiens comme mieux placés qu'une France affaiblie et athée pour préserver ce qui peut encore l'être de la civilisation chrétienne sur place. Du reste, Paris inquiète parce qu'elle ne semble pas s'intéresser suffisamment à l'Indochine, ni y mener une politique cohérente, en phase avec l'évolution des temps. Par ailleurs, si certains observateurs peuvent se montrer rétifs aux réformes en

9. (R. P.) C. Lange, "L'Église catholique au Vietnam ", in C.-R. Ageron (dir.), Les Chemins de la décolonisation de l'empire colonial français (1936-1956), colloque organisé par l'iHtp les 4 et 5 octobre 1984, Paris, CNRS, 1986, p. 179-181. Voir aussi F. Kempf, "Les catholiques français », in M. Merle, (dir.), Les Églises chrétiennes et la décolonisation (Cahiers de la Fondation nationale de sciences politiques, $\mathrm{n}^{\circ}$ 151), Paris, A. Colin, 1967, p. 147-215.

10. On notera par exemple l'influence des évêques locaux sur la ligne éditoriale de Vers l'Avenir (Namur) et de La Gazette de Liège (Radioscopie de la presse belge, Verviers, Marabout, 1975, p. 57-61 et 520-522). Le comité de direction de La Revue nouvelle est composé pour moitié d'ecclésiastiques. Voir J.-L. Jadoulle, Chrétiens modernes? L'engagement des intellectuels catholiques " progressistes " belges de 1945 à 1958 à travers "La Revue nouvelle ", "La Relève » et l'édition belge de "Témoignage Chrétien ", Louvain-la-Neuve, Academia Bruylant-Presses universitaires de Louvain, 2003, p. 49-67.

11. La Revue nouvelle, 15/6/1947, p. 39-51 ; Vers l'Avenir, 18/3/1947, p. 1 et 4.

12. Vers l'Avenir, $7 / 11 / 1947$, p. 3 .

13. Sur l'attitude de ces groupes face à l'Indochine, voir le premier volet de l'étude de S. Rousseau, La Colombe et le napalm. Des chrétiens français contre les guerres d'Indochine et du Vietnam (1945-1975), Paris, CNRs éditions, 2002 ; voir aussi, pour Esprit, M. Winock, "Esprit ", des intellectuels dans la cité (1930-1950), Paris, Seuil, 1996, p. 339-343. 
Afrique, par paternalisme et parce qu'ils sont persuadés d'avoir affaire à des peuples "inférieurs ", ils n'hésitent pas à plaider pour une large autonomie, voire pour l'indépendance en Asie du Sud-Est, région de vieille civilisation que l'on respecte ou que l'on craint.

Fin 1947, en tout cas, les positions de chacun sont fixées. Au fil des mois, il apparaît que les opérations militaires françaises en Asie ne seront pas de courte durée et que l'armée sera contrainte de batailler durablement dans la région. Pourtant, l'Indochine va peu à peu quitter les " unes » des journaux français et devenir " une guerre coloniale oubliée ${ }^{14}$. Cela s'explique notamment parce que la région est lointaine et mal connue, parce que peu d'Européens y vivent, parce que l'opinion est focalisée sur la situation en Métropole et en Europe et parce que le contingent n'est pas sollicité ${ }^{15}$. Les sondages d'opinion le confirment, la France ignore ce qui se passe en Indochine ou y est indifférente - 20 à $30 \%$ des personnes interrogées n'ont pas d'avis sur le sujet $^{16}$-, et les politiques en parlent relativement peu.

En Belgique francophone, on constate, évidemment, les répercussions du phénomène. Patrick Tans, qui a étudié la réception de la guerre d'Indochine dans la presse belge, épingle cependant l'intérêt constant du quotidien catholique bruxellois La Libre Belgique $e^{17}$ et observe que la Flandre s'est sentie moins concernée que Bruxelles et la Wallonie. Il souligne que les journaux et les périodiques belges ont, au total, consacré une très faible surface rédactionnelle au sujet avant la période du dénouement, c'està-dire 1953-1954 ${ }^{18}$.

Du milieu de l'année 1947 à la fin de l'année 1948, on peut même parler d'un réel silence. On évoque bien sûr l'évolution du conflit et les décisions politiques prises à Paris via des dépêches factuelles, mais les articles de fond sont rares. Il faut attendre 1949, avec l'entrée des troupes de Mao à Pékin et la signature des accords Auriol-Bao Daï, faisant de ce dernier le chef de l'État du Vietnam, pour voir naître un regain d'intérêt. Jusqu'au tournant de juin 1950, lorsque commence la guerre de Corée et que les États-Unis réalisent leur première livraison de matériel à l'Indochine, les commentaires de la presse belge francophone, de gauche comme de droite, tournent autour d'une seule et même question : pourquoi ou, plus justement, pour quoi la France se bat-elle en Asie du Sud-Est? Préserve-t-elle

14. J. Dalloz, La Guerre d'Indochine 1945-1954, Paris, Seuil, 1987, titre du chapitre IV, p. 115-158.

15. M. Bodin, "Le moral des militaires français du corps expéditionnaire en Extrême-Orient, 1945-1954 ", in C.-R. Ageron et P. Devillers (dir.), Les Guerres d'Indochine de 1945 à 1975, actes de la table ronde tenue à l'intp les 6-7 février 1995 (Cahiers de l'IHTP, no 34), Paris, CNRs, juin 1996, p. 95-112 ; R. Girardet (dir.), La Crise militaire française (1945-1962). Aspects sociologiques et idéologiques, Paris, A. Colin, 1964, p. 157-172 et Planchais J., Une histoire politique de l'armée. 2. 1940-1967. De de Gaulle à de Gaulle, Paris, Seuil, 1967, p. 199-218.

16. A. Ruscio, «L'opinion française et la guerre d'Indochine (1945-1954). Sondages et témoignages ", in Vingtième siècle. Revue d'histoire, n 29, Paris, janvier-mars 1991, p. 35-45.

17. Par l'intermédiaire de Roman Fajans (1903-1976), ancien grand reporter polonais installé en France après la Seconde Guerre, qui fut le correspondant de La Libre Belgique à Paris de 1946 à 1972.

18. P. Tans, Een vergeten oorlog? De Belgische receptie van de Frans-Indochinese oorlog (1945-1954), mémoire de licence (= maîtrise) en histoire, Katholieke Universiteit Leuven (KUL), 1986. 
encore son propre intérêt, et notamment sa puissance, ou défend-elle, volontairement ou non, une cause plus large ? L'orientation de plus en plus nette d'Hô Chi Minh vers Moscou et Pékin rend chaque jour plus pertinente l'interrogation autour du contenu idéologique et stratégique de la guerre d'Indochine.

Ce contexte particulier va renforcer l'extrême-gauche communiste dans son soutien à la cause Vietminh, maintenir les socialistes dans une inconfortable hésitation - même si les journaux les moins organiquement liés au parti vont se faire de plus en plus antifrançais -, conforter la position antiVietminh des libéraux et amener une évolution du côté catholique. Le monde chrétien est désormais pris entre deux feux : prôner l'émancipation vietnamienne au profit de l'Église est une chose, mais risquer de le faire au profit du communisme en est une autre. Le cas du R. P. Lozet en témoigne clairement : il poursuit certes sa croisade contre la France révolutionnaire, la France païenne qui a voulu assimiler des populations indigènes en niant leurs spécificités et a simplement réussi à exporter ses tensions, mais il reconnaît que la poussée communiste contraint désormais la France - mais une France chrétienne, espèret-il - à demeurer en Indochine ${ }^{19}$.

\section{L'AMBASSADE DE FRANCE À BRUXELLES \\ ET LE DÉCLENCHEMENT DU CONFLIT INDOCHINOIS : PARER LES PREMIERS COUPS}

L'exposé des faits en témoigne : la guerre d'Indochine place Paris devant une double contre-propagande en Belgique. Les attaques de la gauche y sont épaulées par celles d'un monde catholique qui voit sa survie outre-mer dans la dissociation claire entre colonisation et christianisation. Pour la France officielle, jouer la carte, hier payante, du bilan civilisateur est désormais un stade révolu, même si l'on essaie encore de "vendre " ce thème, par le biais de conférenciers ou par le placement d'articles - les Pages de France ${ }^{20}$ dans l'un ou l'autre périodique favorable.

Aux premiers jours du conflit, c'est surtout de gauche que vient le danger. Le Drapeau rouge et Le Peuple ne ménagent pas leurs critiques à l'égard de la France. Le quotidien communiste rend ainsi les milieux réactionnaires français responsables de l'insurrection par leur sabotage systématique des accords passés en vue de l'indépendance vietnamienne ${ }^{21}$. Très irrité,

19. Revue nouvelle, 7-8/1950, p. 105-112.

20. La diffusion des Pages de France-Extinfor est l'une des missions du Service d'action culturelle à l'étranger ou Service des échanges culturels, au sein de la DG Relations culturelles du Quai d'Orsay. Voir F. Roche et B. Piniau, Histoires de diplomatie culturelle des origines à 1995, Paris, Ministère des Affaires étrangères - ADPF/La Documentation française, 1995, p. 77 et sq. ; R. Frank, "La machine diplomatique culturelle française au $\mathrm{xx}^{\mathrm{e}}$ siècle ", in Relations Internationales, $\mathrm{n}^{\circ} 115$ (Diplomatie et transferts culturels au $\mathrm{XX}^{\mathrm{e}}$ siècle - 1), Paris, automne 2003, p. 330.

21. Drapeau ronge, $21 / 12 / 1946$, p. 1. 
l'Ambassadeur de France à Bruxelles, Raymond Brugère ${ }^{22}$, parle d'articles " parfaitement tendancieux " et y voit une offensive synchronisée de Moscou ainsi que l'application de consignes soviétiques à des fins idéologiques, puisque le sujet n'intéresse pas directement l'opinion belge ${ }^{23}$. Par ailleurs, le diplomate soupçonne également une manœuvre de L'Humanité. Le PCF étant alors au Gouvernement, son quotidien officiel réfrénerait ses ardeurs sur la question indochinoise, mais se servirait de son homologue belge comme exutoire. Il s'y publie [dans Le Drapeau Rouge] un article quotidien en faveur du Vietnam. Il est impossible de supposer qu'une telle campagne soit d'inspiration belge ; il y a tout lieu de penser que L'Humanité, faute de vouloir les utiliser elle-même en ce moment, passe par téléphone à sa réplique communiste de Bruxelles, l'essentiel des allégations l'alimentant ${ }^{24}$.

Autre sujet de préoccupation pour Brugère : certains papiers signés, dans Le Peuple, par Grégoire Koulischer ${ }^{25}$. Le 20 décembre 1946, le chroniqueur de politique étrangère titre ainsi «Vers l'indépendance des empires coloniaux $»^{26}$. La semaine suivante, il s'attache plus précisément à la situation indochinoise. Jugeant close l'ère de la colonisation, il insiste sur le caractère irrésistible et irréversible d'un processus dont les socialistes anglais et français, alors au pouvoir, doivent faciliter le déroulement pacifique. Le texte de Koulischer est un appel clair au très temporaire gouvernement SFIO homogène mené par Léon Blum : il s'agit de « renverser la vapeur et de liquider l'héritage des gouvernements impérialistes de jadis ". Mais, au-delà de ces considérations politiques, le journaliste s'attarde sur les méthodes de guerre employées par la France. Alors que les Vietnamiens alignent "quelques Japonais " ayant échappé à la vigilance des Alliés, il note que la France emploie « des Allemands, anciens soldats

22. Fils d'un ancien généralissime, Raymond Brugère (1885-1966) entra dans la carrière diplomatique au lendemain de la Première Guerre et fut notamment conseiller d'ambassade à Bruxelles auprès de Paul Claudel avant de devenir ministre plénipotentiaire en 1934. Le 17 juin 1940, alors qu'il était en poste à Belgrade, il rendit sa démission, désapprouvant la demande d'Armistice formulée par Pétain. Mis à la retraite d'office, il entra dans la Résistance, tenta de gagner Londres, mais finit par être arrêté et interné de novembre 1942 au 8 juin 1944. Gaulliste fervent, il fut nommé, le 21 septembre 1944, secrétaire général du Quai d'Orsay puis, dès le 4 octobre, ambassadeur en Belgique. Suite à un violent désaccord avec Georges Bidault, il fut définitivement admis à la retraite fin 1947, mais s'empressa de rejoindre le tout jeune RPF du général de Gaulle dont il présida la commission des Affaires étrangères. Sur lui, voir notamment J.-B. Duroselle, L'Abîme 1939-1944 (Politique étrangère de la France 1871-1969), Paris, Imprimerie nationale, 1982, p. 187 et ses souvenirs : Veni, vidi, Vichy... et la suite. Témoignages (1940-1945), Paris, Les Deux-Rives, 1953.

23. Archives du ministère des Affaires étrangères français-Centre des archives diplomatiques de Nantes-Fonds ambassade de France à Bruxelles. Série D : 1930-1959 (désormais AMAEF-A), dossier 15, sous-dossier France d'outre-mer 1944-1957, télégramme de Brugère, 21/12/1946.

24. Idem, 27/12/1946.

25. Docteur en sciences politiques, Gricha dit Grégoire Koulischer (1908-1991) quitta Kiev au moment de la révolution bolchevique et s'installa en Belgique en 1925. Journaliste au Peuple dès 1935, il fut en charge des nouvelles parlementaires puis de la politique étrangère. Durant la Seconde Guerre, il gagna le Canada puis Londres, où il œuvra au sein du Political Intelligence Department. Officier au Service de presse de la Mission militaire belge en 1944-1945 puis attaché au cabinet du Premier ministre Van Acker, il resta chroniqueur de politique étrangère au Peuple jusqu'en 1948.

26. Peuple, 20-21/12/1946, p. 3. 
de l'Afrika-Korps de Rommel, faits prisonniers en Afrique et engagés à la Légion étrangère ». Ordre leur serait donné d'être particulièrement durs avec les indigènes parlant le français, d'autant plus dangereux qu'ils sont instruits ${ }^{27}$.

Aux yeux de Raymond Brugère, cet article du Peuple est " encore plus déplaisant et malveillant " que ceux du Drapeau ronge. Outré, il minimise pourtant son impact : en Belgique, nul ne pourra croire que ce sont les Français qui se conduisent en barbares. Néanmoins, l'ambassadeur est décidé à se plaindre aux dirigeants socialistes et communistes belges « de l'usage que certains de leurs sous-ordres croient devoir faire de pareilles accusations, vinssent-elles de Paris ». Il annonce également au Quai d'Orsay qu'il contactera le ministre socialiste des Affaires étrangères, Paul-Henri Spaak, et qu'il a déjà évoqué la question avec Victor Larock, le directeur du Peuple. Embarrassé, celui-ci l'a assuré que l'article lui avait échappé, qu'il pouvait avoir été inspiré par une infirmière française revenue mécontente d'Indochine et que les instructions voulues allaient être données pour que le journal ne laisse plus passer d'informations aussi malveillantes ${ }^{28}$.

De fait, alors que l'anticolonialisme de gauche et d'extrême gauche continuera à prospérer, le moniteur officiel du PSB se montrera très prudent en ce qui concerne l'Indochine, adoptant une neutralité souvent bienveillante envers la France. Fin 1948, Koulischer lui-même quitte Le Peuple pour diriger le service de presse du Bureau international du travail. Il faut attendre la fin de l'année 1950 pour voir l'ambassade relever, de nouveau, un article perçu comme "partisan » dans la mesure où il met en parallèle la réussite du Gouvernement travailliste anglais en Inde et les échecs de la politique française en Indochine ${ }^{29}$.

Si Brugère doit canaliser la presse de gauche, il peut compter sur le soutien du centre et d'une partie de la droite. L'ambassadeur a ses entrées au Face à Main, hebdomadaire illustré bruxellois de tendance libérale qui, début 1947, fait massivement la publicité de l'œuvre française en Indochine. Le numéro du 4 janvier livre un portrait plus que flatteur de l'amiral d'Argenlieu ${ }^{30}$, tandis que deux pages de clichés vantent les efforts de la France en matière de préservation des sites indigènes et de scolarisation des jeunes indochinois ${ }^{31}$. De la même manière, le 21 janvier 1947, le quotidien conservateur bruxellois La Nation belge publie en bonne place une Page de France signée

27. Idem, 27/12/1946, p. 3. Sur le poids de ces légionnaires allemands en Indochine dans la propagande antifrançaise au cœur des deux Allemagnes, voir C. Metzger, "L'opinion publique est-et ouest-allemande face à la décolonisation en Indochine (1949-1954) ", in Guerres mondiales et conflits contemporains, $\mathrm{n}^{\circ} 164$, octobre 1991, p. 93-113.

28. AMAEF-A, dossier 15, sous-dossier France d'uutre-mer 1944-1957, télégramme de Brugère, 27/12/1946.

29. Idem, de de Hauteclocque à Schuman, 21/11/1950 concernant Le Peuple, 16/11/1950, p. 1.

30. Face à Main, 4/1/1947, couverture et p. 3 .

31. Idem, pages centrales. 
Edmond Delage ${ }^{32}$ et intitulée : "Ce que Paris peut opposer aux revendications du Vietnam. La France a fait de l'Indochine un État moderne et son action y fut bienfaisante ${ }^{33}$. À l'entame du conflit, on voit donc l'ambassade de France utiliser ses divers canaux de propagande pour faire pièce à l'anticolonialisme d'une partie de la gauche belge.

\section{LES ATTAQUES DU MONDE CATHOLIQUE :}

L'AFFAIRE JACQUES-HOUSSA-KUU

Du côté catholique, l'attention de l'ambassade est très tôt attirée par l'activité d'un franciscain belge, missionnaire en Indochine, le R.P. Emmanuel Jacques-Houssa. Début juillet 1946 déjà, Alphonse Clarac, conseiller diplomatique du haut-commissaire pour l'Indochine d'Argenlieu, fait parvenir au Quai d'Orsay et au ministère de la France d'outremer un rapport sur ses "activités antifrançaises ». Au moment où il allait embarquer pour la France, on a saisi sur lui des documents dénonçant « la crise des catholiques annamites ". Houssa transportait ainsi une lettre de $M^{\mathrm{gr}}$ Lê Huu Thu, vicaire apostolique de Phat Diem, adressée au recteur de l'université de Louvain et affirmant ouvertement que l'ouverture d'une université catholique dirigée par des professeurs non-Français rendrait des services à la cause chrétienne en Asie. Houssa ramenait également avec lui des brochures rédigées par des prêtres vietnamiens se disant opprimés par leurs homologues français. Identifiant les doctrines chrétiennes avec le nationalisme et l'apostolat missionnaire français avec le colonialisme, ils en appellent à une Église vietnamienne autonome. Pour le haut-commissariat, l'affaire est grave et nécessite à tout le moins qu'on interdise à Houssa un retour au Vietnam ${ }^{34}$.

$\mathrm{Au}$ sein du catholicisme vietnamien, Lê Huu Thu et le Phat Diem représentent un élément de poids. Situé au sud du delta du fleuve Rouge, à la limite du Tonkin et de l'Annam, l'évêché, fort de 500 paroisses, est le premier secteur totalement confié à un clergé d'origine locale. Le vicaire apostolique Lê Huu Thu est aussi, dans un premier temps, conseiller de Hô Chi Minh. Avec l'appui de celui-ci, il constitue une zone autonome dans laquelle il lève l'impôt, organise la milice, bref règne presque comme le chef d'une principauté qui reconnait la seule autorité du Vatican. Très vite pourtant, Lê Huu Thu se positionnera à la fois contre la France et contre le communisme Vietminh, entendant rester maître d'une enclave

32. Normalien et agrégé d'allemand, Edmond Delage (1886-1968) enseigna dans plusieurs écoles militaires de haut niveau. Vice-président de l'Académie de marine et vice-président délégué de la Ligue maritime d'outre-mer, il fut chef du Service de documentation à la section histoire de l'étatmajor de la Marine. Chroniqueur au Monde, critique musical à La Libre Belgique, il vit ses articles publiés dans de nombreux organes de presse français et belges.

33. Nation Belge, $21 / 1 / 1947$, p. 2.

34. AMAEF-A, dossier 15, sous-dossier France d'outre-mer 1944-1957, du haut-commissaire de France pour l'Indochine au ministère des Affaires étrangères, 8/7/1946. 
nationaliste et catholique. Fin 1947, il sera lâché par Hô Chi Minh qui exercera une pression de plus en plus forte sur le Phat Diem. En 1949, la région acceptera l'occupation militaire française et se rallie officiellement à la solution Bao Daï ${ }^{35}$. Néanmoins, la politique de Lê Huu Thu restera "personnelle et sinueuse $»^{36}$.

Au moment où le R. P. Houssa est arrêté, le clergé vietnamien est donc considéré par la France comme hostile, ce qui explique la dépêche angoissée du haut-commissariat. Dans les mois qui suivent, on perd pourtant la trace du missionnaire belge, de l'aveu même de Frank Puaux, consul de France à Gand et Courtrai, mais, de source sûre, il serait à Louvain fin décembre $1946^{37}$. C'est à ce moment qu'il fait paraître, dans La Revue nouvelle, très proche des milieux louvanistes, une étude sur la "Situation du catholicisme au Vietnam $»^{38}$. Il y explique d'abord que la colonisation européenne a entraîné un grand nombre de bouleversements en Indochine avec l'introduction de l'individualisme et du machinisme. Les conflits philosophiques et sociaux ont été compliqués à ses yeux «par la présence des Occidentaux, et principalement des Français, car si tous les Vietnamiens ne participaient pas à la lutte clandestine, bien rares étaient ceux qui préféraient l'occupation étrangère à l'indépendance " ${ }^{39}$. De là découle l'échec partiel du christianisme qui est avant tout, écrit Houssa, l'échec des Européens chrétiens dans la mesure même où l'évangélisation s'est confondue avec la colonisation, au profit de cette dernière ${ }^{40}$. Le missionnaire belge met l'Europe en accusation : "par elle, dit-il, le Christ a été trahi et presque vendu " puisqu'elle a négligé la formation théologique et profane de ses missionnaires avec pour conséquence d'avoir donné un niveau intellectuel plus que médiocre au clergé indigène ${ }^{41}$. Dès lors, cette négligence, cette faillite ont laissé le champ libre au communisme ${ }^{42}$. Pour Houssa, le seul espoir réside dans le positionnement ferme des quatre évêques vietnamiens et de leurs fidèles en faveur de l'indépendance, positionnement qui "a effacé aux yeux des hommes de bonne foi le souvenir des compromissions passées avec le pouvoir étranger ${ }^{43}$. Il s'agit donc d'une charge à fond contre la France et sa politique que La Revue nouvelle publie sans commentaires.

Un mois plus tard, un autre organe catholique, La Gazette de Liège, donne la parole, en "une ", à " un Belge qui connaît parfaitement " le Vietnam, mais dont elle ne cite pas le nom. Il est toutefois plausible qu'il s'agisse d'Emmanuel Jacques-Houssa. Celui-ci diffuse, de nouveau, son

35. C. Lange (R. P.), «L'Église catholique au Vietnam »..., p. 186-187.

36. J. Dalloz, La Guerre d'Indochine..., p. 153.

37. AMAEF-A, dossier 15, sous-dossier France d'outre-mer 1944-1957, sous-dossier affaire HoussaKuu, de Puaux à Brugère, 16/12/1946.

38. Revue nouvelle, 15/12/1946, p. 765-775.

39. Idem, p. 767.

40. Idem, p. 768 .

41. Idem, p. 769.

42. Idem, p. 770-771.

43. Idem, p. 773. 
message : les aspirations vietnamiennes à l'indépendance ne viennent ni de l'envahisseur japonais, ni des communistes mais existent depuis de longues décennies. Il poursuit en accusant la France d'avoir avancé la défense des missions comme un prétexte pour mieux s'implanter de manière lucrative, d'avoir accaparé la souveraineté, d'avoir multiplié les fonctionnaires importés puis, peu à peu, d'avoir exporté son laïcisme en Asie. Les catholiques vietnamiens souffrent, comme les non-catholiques, d'être infériorisés, conclut le témoin. Joseph Demarteau, le patron de La Gazette de Liège, semble se rallier au constat et justifie l'ouverture de ses colonnes à une thèse controversée dans la mesure où l'AFP, très contrôlée par le Gouvernement de la République, verrouille tout ce qui ressemble, de près ou de loin, à une contestation de la politique française ${ }^{44}$. Il s'agit donc, argue-t-il, de diversifier les sources d'information.

Poursuivant dans cette logique, le quotidien liégeois multiplie, dans les semaines qui suivent, les articles et les correspondances particulières favorables aux indépendantistes vietnamiens. Mi-février, trois papiers, signés d'un ancien professeur en Indochine, expliquent comment et pourquoi, au-delà des brimades et des condamnations politiques injustes, les Vietnamiens continuent à se battre pour leur nation occupée ${ }^{45}$. Deux mois plus tard, le même s'insurge contre les informations tronquées que diffuse la grande presse. Influencée par la France, celle-ci met l'accent sur la violence des insurgés alors qu'elle néglige, dit-il, "les procédés raffinés ou la brutalité plus élémentaire de trop de coloniaux ». L'auteur réclame le silence mutuel puisqu'il y a des tueurs dans chaque camp. Plus encore, s'il ne justifie pas l'action des Vietnamiens, il la compare à celle de la résistance belge durant la Seconde Guerre toute proche. Évoquant ses anciens élèves, ses anciens scouts qui combattent dans le maquis indochinois, il dit souffrir de les voir condamnés en bloc pour les excès de quelques-uns, tout comme, lance-t-il habilement, on accuse à tort la France entière parce qu'elle compte des Allemands, voire d'anciens ss dans les rangs de sa Légion étrangère ${ }^{46}$. Le fait a beau être excusé, il n'en est pas moins souligné.

Soutenu par La Gazette de Liège, Emmanuel Jacques-Houssa l'est aussi par La Libre Belgique qui, le 26 février 1947, ouvre à son tour sa " une " au récit de ses souvenirs du Vietnam. Le religieux y distribue les bons et les mauvais points: les Vietnamiens sont décrits comme nationalistes, mais non xénophobes, ils sont assimilés aux résistants même si Houssa reconnaît que certains d'entre eux commettent des atrocités injustifiables, au même titre que la police française. Plus modéré qu'à l'accoutumée, l'auteur conclut que le bilan de la colonisation française est contrasté, mais que le fléau de la balance penche plutôt du côté positif ${ }^{47}$. Sans doute a-t-il

\footnotetext{
44. Gazette de Liège, 16/1/1947, p. 1.

45. Idem, du 11 au 13/2/1947.

46. Idem, $14 / 4 / 1947$, p. $1-3$.

47. Libre Belgique, 26/2/1947, p. 1.
} 
compris que le lectorat de La Libre Belgique, catholique mais bourgeois et conservateur, risquait de ne pas entendre un message aux accents trop anticolonialistes et révolutionnaires.

À la lecture de son témoignage, l'ambassade ne manque pas de réagir. Raymond Brugère observe que la gauche, qui a provisoirement cessé sa propagande, est désormais relayée par la droite. Mais, il se veut rassurant : "de telles absurdités dans un journal connu pour être architendancieux sont de nature à nous faire plus de bien que de mal ", estime-t-il avant de mettre le témoignage d'Houssa en parallèle avec certains articles de Roman Fajans sur un prétendu réveil du nazisme français ${ }^{48}$. Le tout donne l'impression au diplomate d'un "imbroglio de menées antifrançaises " qui pourrait être inspiré par certains milieux français de droite et répercuté ensuite par leurs relais à l'étranger ${ }^{49}$. L'idée d'une contre-propagande organisée est donc bien présente dans l'esprit de Brugère.

Elle l'est d'autant plus qu'Emmanuel Jacques-Houssa s'est lancé, avec un Vietnamien du nom de Truong-Long dit Vincent Kuu, dans une tournée de conférences. Certes, les tribunes qui les accueillent sont relativement confidentielles, mais l'ambassade n'en est pas moins inquiète. Le 11 février 1947, Kuu parle à l'Union des étudiants catholiques de Liège en tant que secrétaire de la Fédération des Vietnamiens catholiques de France. Le consul général de France à Liège, Jules Daniel-Lamazière, rapporte que l'homme se donne pour docteur en droit et en sciences politiques et sociales de l'université de Paris et qu'il a cherché, durant son séjour à Liège, à entrer en contact avec divers milieux catholiques en demandant aux familles d'aider les étudiants annamites de France, souvent dans le dénuement. Durant son exposé, il s'est livré à des attaques contre la France et à une glorification du soulèvement vietnamien, ce qui a amené des incidents avec une partie du public et a contraint le président à lever la séance dans la mesure où Kuu n'avait pas respecté son engagement d'objectivité ${ }^{50}$.

Nullement découragé, Kuu est à Gand deux jours plus tard, à l'Association catholique d'aide aux missions. Le consul Frank Puaux, qui le suit à la trace, note qu'il s'y est exprimé devant un auditoire très clairsemé - quatorze personnes - mais qu'il parlera à Bruxelles le 23 février, sous l'égide de la Jeunesse ouvrière chrétienne ${ }^{51}$. Dans la capitale, ce sont les services de Brugère qui prennent le relais de la surveillance. Le soir prévu, ils sont dans la salle, avec cent cinquante personnes, surtout des étudiants, une vingtaine de prêtres et des membres de la JOc. Mais, Kuu n'arrive pas, bloqué à la frontière par les douaniers pour insuffisance de pièces d'identité. C'est alors Houssa qui le remplace au pied levé, se livrant à des considérations générales sur les mœurs et la civilisation vietnamiennes. L'abbé Bourguignon,

48. Ibidem : Le nazisme français n'est pas mort.

49. AMAEF-A, dossier 15, sous-dossier France d'outre-mer 1944-1957, sous-dossier affaire HoussaKuu, télégramme de Brugère, 28/2/1947.

50. Idem, de Daniel-Lamazière à Brugère, 22/2/1947.

51. Idem, de Puaux à Brugère, 14/2/1947. 
pour la JOC, clôture la soirée en soulignant l'existence en Belgique d'Amitiés vietnamiennes qui possèdent des foyers dans les principales villes du pays. L'ambassade s'interroge évidemment sur cette association et en arrive à la conclusion qu'elle implique ouvertement le clergé de Louvain, coupable de soutenir un mouvement qui "cache une activité sourde contre notre influence en Indochine ». Mais, au-delà, c'est Houssa qui est dans la ligne de mire comme principal organisateur et recruteur de conférenciers dans les milieux annamites de Paris ${ }^{52}$.

À la fin du mois de février, Vincent Kuu est de retour dans la province de Liège où il semble disposer de nombreux soutiens. Le 24, il devait parler au Collège jésuite Saint-François-Xavier de Verviers devant cent cinquante personnes mais, de nouveau, un problème de visa l'empêche de franchir la frontière. De toute évidence, Paris met un point d'honneur à ce qu'il ne s'exporte pas. Comme à Bruxelles, c'est Houssa qui prend la parole à sa place, n'hésitant pas, cette fois, à se faire virulent. Pour le consul Daniel-Lamazière, ce sont les jésuites qui favorisent en Belgique la propagande provietnamienne et ont notamment obtenu, sur Verviers, l'annonce de la conférence dans le quotidien catholique de droite Le Courrier du Soir, malgré les récriminations de nombreux milieux chrétiens locaux ${ }^{53}$.

Mais, Kuu persiste. Le 24 mars 1947, il est annoncé au Cercle catholique Sainte-Marguerite, à Liège ${ }^{54}$, puis, mi-avril, parle de nouveau à l'Union des étudiants catholiques où cette seconde soirée se déroule sans accroc. La Gazette de Liège lui accorde un compte-rendu et relaie l'appel du conférencier aux familles catholiques liégeoises, les engageant à accueillir des étudiants vietnamiens. Selon le quotidien, la séance s'est terminée sous les applaudissements du public ${ }^{55}$.

On perd ensuite la trace de Kuu, qui semble mettre une sourdine à sa campagne de propagande en Belgique. Houssa, pour sa part, a gagné les États-Unis, où il s'emploie à obtenir des bourses pour les étudiants vietnamiens au sein de collèges et universités catholiques ${ }^{56}$. Toutefois, leur agitation de 1947 a secoué Paris : il faudrait qu'aux attaques contre la position de la France en Indochine réponde une propagande officielle rapide, ciblée et efficace. Or, on est loin du compte...

\section{UNE PROPAGANDE FRANÇAISE TROP POUSSIVE (1947-1950)}

L'impression dominante est en effet celle d'une France impuissante à réagir ou se consacrant à une simple propagande défensive. Ainsi, en juin 1947, le Bulletin hebdomadaire de presse de l'ambassade relève que

52. Idem, de Brugère à Bidault, 25/2/1947.

53. Idem, de Daniel-Lamazière à Bidault, 3/3/1947.

54. Idem, de Daniel-Lamazière à Teitgen, ministre ad interim, 24/3/1947.

55. Gazette de Liège, 15/4/1947, p. 3.

56. D. H. Nguyêñ, From the City inside the Red River: a Cultural Memoir of Mid-Century Vietnam, Jefferson, McFarland, 1999, p. 141. 
La Métropole, quotidien catholique francophone anversois, accorde "une importance et une créance fâcheuses aux renseignements fournis par les "services d'information vietnamiens ", ce qui lui fait annoncer officiellement que deux cents Marocains et Malgaches se sont refusés à combattre en Indochine ${ }^{57}$. Ceci constaté, l'ambassade ne va guère plus loin. Elle se contente de qualifier l'article de "profondément regrettable ", mais n'annonce aucune démarche précise, aucune tentative pour obtenir un rectificatif comme si, sur ce terrain, elle partait déjà perdante ${ }^{58}$.

Dans d'autres cas, le poste de Bruxelles souhaite contre-attaquer, mais se heurte à l'inertie, volontaire ou non, de Paris. Un exemple frappant est offert en février 1950, alors que la situation sur le terrain s'est profondément modifiée. Hô Chi Minh vient d'être reconnu par la toute jeune République populaire de Chine et par l'urss. Du côté occidental, la France vient de ratifier les accords de 1949 qui rendent effective la souveraineté du Vietnam, sous l'autorité de Bao Daï et dans le cadre de l'Union française, mais elle est surtout aux prises avec une agitation communiste de plus en plus forte contre la "sale guerre " et avec les soubresauts de "l'affaire des généraux ", scandale d'espionnage au profit des communistes qui mouille de hauts gradés français et sur lequel se greffe l'affaire du trafic de piastres ${ }^{59}$.

Le 4 février 1950, La Libre Belgique publie en bonne place une carte de l'Indochine sous le titre Le Nouveau Front soviétique de la guerre froide. Y sont teintés de noir tous les territoires constituant alors le Vietnam et que le quotidien catholique présente comme étant sous la domination d'Hô Chi $\operatorname{Minh}^{60}$. À l'ambassade, on s'insurge contre un document «non seulement tendancieux mais outrageusement faux et de nature à tromper gravement l'opinion publique belge ». On note aussi que la carte, sans doute diffusée par une agence, a également été publiée le 6 février dans Le Matin, quotidien libéral d'Anvers, ainsi que dans La Meuse et La Lanterne, deux journaux d'information appartenant au même groupe. Toutefois, dans le premier cas, elle était qualifiée de "manœuvre soviétique " et, dans le second, elle venait illustrer un article du reporter français Lucien Bodard ${ }^{61}$ mettant en valeur le recul du Vietminh. C'est donc uniquement sur La Libre Belgique qu'il faut faire porter l'effort ${ }^{62}$.

Le général de Lattre, qui commande les forces françaises en Indochine, est alors présent à Bruxelles. Alarmé par la carte litigieuse, il réclame de la

57. Métropole, 12/6/1947, p. 1 .

58. AMAEF-La Courneuve-Fonds direction politique, Z-Europe, Belgique 1944-1949, dossier 10, Bulletin hebdomadaire de presse $\mathrm{n}^{\circ} 131,13 / 6 / 1947$, p. 5-6.

59. Sur ces affaires: P. Marcus, La République trahie. L'affaire des généraux-l'affaire des piastresl'affaire des fuites, Paris, Le Cherche-Midi, 2009 ; Renaud-Groison J., Complots en France, Paris, Picollec, 1980.

60. Libre Belgique, 4/2/1950, p. 3.

61. Fils du consul de France à Tchoung-King, Lucien Bodard (1914-1998) passa son enfance en Asie. Il fut le correspondant de guerre de France-Soir en Indochine durant la majeure partie du conflit et demeura en Extrême-Orient jusqu'en 1960, avant de couvrir la guerre d'Algérie.

62. AMAEF-A, dossier 15, sous-dossier France d'outre-mer 1944-1957, de Hauteclocque au ministère des Affaires étrangères, 6/2/1950. 
Défense nationale l'envoi d'une carte correspondant réellement à la situation pour pouvoir contre-attaquer. Néanmoins, le ministère ne donne pas suite, ce dont l'ambassadeur Jean de Hauteclocque ${ }^{63}$ se plaint amèrement au Quai d'Orsay. Un collaborateur du diplomate est alors chargé de faire une démarche directement auprès de La Libre Belgique, en insistant non sur les répercussions négatives pour l'image de la France, argument peu porteur aux yeux d'un organe perçu comme francophobe, mais sur l'appui indirect apporté à la propagande communiste. De Hauteclocque rapporte que le journal a "courtoisement » reconnu sa maladresse et s'est dit prêt à la rectifier dès que possible. Intervient ailleurs un second cafouillage dans le système français : pour l'ambassadeur, l'affaire aurait déjà été bouclée si le service de presse du poste disposait d'une documentation suffisante, telle celle fournie aux revues françaises. En son absence, il faut parer au plus pressé et de Hauteclocque suggère au Quai d'Orsay de convoquer Roman Fajans pour le renseigner sur la question indochinoise et lui demander de rédiger une correspondance ${ }^{64}$.

L'ambassade de France dégage donc toutes les possibilités de réaction et tente de réveiller une administration centrale manifestement peu pressée de répliquer aux attaques psychologiques de l'adversaire. Par ailleurs, quand réaction il y a, celle-ci ne suit pas forcément les canaux prévus, ce qui induit un sentiment d'improvisation. Le 25 février 1950, soit plus de vingt jours après la publication de la carte, Jean de Hauteclocque adresse une dépêche très ferme à son ministre de tutelle, Robert Schuman, pour se plaindre des pratiques en vigueur au ministère de la France d'outre-mer, géré alors par un autre MRP, Jean Letourneau. Ayant reçu du Service d'information de ce ministère la copie d'une rectification directement adressée à La Libre Belgique, l'ambassadeur s'enflamme : "Je voudrais que vous lui fassiez savoir qu'il n'est pas seulement incorrect mais aussi imprudent de transmettre des communications à un journal étranger sans passer par le chef du poste diplomatique ; incorrect parce qu'il a seul la charge et la responsabilité des communications officielles [...] françaises et imprudent parce que, s'agissant d'un journal comme La Libre Belgique, toute communication écrite peut donner lieu à des commentaires malveillants et tendancieux auxquels ce journal nous a particulièrement habitués». Et d'ajouter que, si on lui avait simplement envoyé la communication, il se serait contenté d'une démarche verbale, "à tous points de vue plus opportune ${ }^{65}$, entendez plus discrète et moins dirigiste.

63. Cousin du maréchal Leclerc, Jean de Hauteclocque (1893-1957) fut d'abord officier mais, gravement blessé durant la Première Guerre, il opta pour la diplomatie. Démissionnaire en 1941, il se montra bientôt actif dans la Résistance. Arrêté par la Milice en mars 1944, il s'échappa et refit surface à la Libération. Il fut alors brièvement délégué du Gouvernement provisoire en Belgique puis ambassadeur à Ottawa avant de retrouver Bruxelles (1947-1951). Résident général en Tunisie (1952-1953), ambassadeur à Lisbonne (1955-1956) puis conseiller diplomatique du Gouvernement, il fut élu maire dans le Pas-de-Calais en 1957 mais décéda la même année.

64. AMAEF-A, dossier 15, sous-dossier France d'outre-mer 1944-1957, de de Hauteclocque au ministère des Affaires étrangères, 6/2/1950.

65. Idem, 25/2/1950. 
Quoi qu'il en soit, l'ambassade attend toujours qu'on lui fournisse la documentation susceptible de rectifier, en cas de besoin, les assertions perçues comme nuisibles à la cause française en Indochine. Il faut attendre la fin du mois de mars 1950, soit près de six semaines, pour que l'ambassade reçoive une circulaire officielle avec carte ainsi qu'un numéro récent des Notes et études documentaires de "La Documentation française " sur le nouveau statut juridique de l'Indochine. Conscient de son retard, le ministère des Affaires étrangères demande à Jean de Hauteclocque de mettre néanmoins tout en œuvre pour en obtenir la publication, en tout ou partie, dans la presse belge ${ }^{66}$.

En attendant, pendant que Paris tergiverse et que l'ambassade s'émeut, La Libre Belgique continue, elle, de privilégier, sur la situation indochinoise, des sources d'information non françaises. Début mai 1950, elle publie ainsi, en deux volets ${ }^{67}$, le récit d'un reporter américain, transmis par l'agence AP. L'homme est présenté comme ayant passé quatre ans au Vietnam, ce qui, précise-t-on toutefois, n'est pas un gage d'objectivité. Loin de montrer la guerre d'Indochine comme un pan de la guerre froide, le correspondant explique que les Français combattent pour protéger sur place leurs capitaux, leurs investissements, une source permanente d'importants revenus, mais aussi le prestige qui leur permet de ne pas devenir une puissance de troisième zone ${ }^{68}$. Quelques semaines plus tard, le déclenchement de la guerre de Corée va, certes, contribuer à modifier la perception américaine du conflit et, par ricochet, celle de La Libre Belgique. Il n'en reste pas moins que l'image renvoyée ici de la France est celle, peu flatteuse, d'une nation en déclin, arc-boutée sur ses intérêts matériels immédiats.

Quelles conclusions tirer au terme de cette première phase du conflit indochinois ? L'analyse rationnelle de la propagande française en Belgique contraint à dresser un bilan plutôt négatif. Paris s'est trouvée attaquée sur deux fronts : si les critiques de l'extrême gauche étaient attendues et ont eu peu d'impact en dehors de cette sphère de l'opinion, celles du monde catholique dérangent davantage, dans un pays où l'Église possède encore à ce moment un pouvoir d'influence non négligeable. Face à cette opposition multiforme, la France n'a que partiellement réussi à transmettre son message, grâce à la complicité de certains "faiseurs d'opinion » acquis à sa cause ou grâce à certaines pressions exercées auprès des Affaires étrangères belges. L'ambassade de France à Bruxelles a, par ailleurs, rapidement dénoncé un suivi défaillant du dossier à Paris.

66. Idem, du ministère des Affaires étrangères à l'ambassade, 24/3/1950.

67. Libre Belgique, 5 et $10 / 5 / 1950$.

68. Idem, 10/5/1950, p. 5 . 
En bref, la France a souvent manqué de réactivité et n'a jamais réellement adopté une attitude proactive. Considérait-on déjà la partie comme perdue ? A-t-on suffisamment pris la mesure du changement majeur que la Seconde Guerre a induit dans les canaux d'information des Belges francophones? Dans les années trente, l'agence de presse Belga était alimentée à $80 \%$ par Havas ${ }^{69}$. Depuis 1945 , Belga est abonnée à une série d'agences internationales parmi lesquelles l'AFP et la britannique Reuter sont certes dominantes, mais le quasi, monopole français a cessé. En outre, certains journaux ont un abonnement direct aux agences américaines AP Ou $\mathrm{UPI}^{70}$, ce qui, dans le cas de l'Indochine, ouvre à une propagande anti-française manifeste. D'autre part, si le Belge francophone lit encore la presse française, aucune comparaison n'est possible avec la diffusion massive des quotidiens parisiens dans les années trente. 100 à 120000 journaux français étaient écoulés quotidiennement en Belgique en 1939, mais ce chiffre est tombé à 10000 en $1946^{71}$. Il faudra attendre 1949 pour voir la situation s'améliorer pour les magazines, mais guère pour les quotidiens. En d'autres termes, il est désormais beaucoup plus difficile pour la France de peser, directement ou non, sur l'opinion belge de langue française. Le cas de la guerre d'Indochine n'est qu'un exemple parmi d'autres, mais un exemple frappant dont la France saura partiellement se souvenir lors de la guerre d'Algérie. Pour ce conflit plus visible, plus " médiatique », Paris se heurtera à une contre-propagande d'une férocité accrue, mais se montrera néanmoins plus combative et inventive pour la contrer.

Catherine LANNEAU Université de Liège

69. R. Demoulin, "Comment un diplomate français voyait la presse belge à la veille de la Seconde Guerre mondiale ", in Revue du Nord (t. LXVI, n ${ }^{\text {os } 261-262), ~ L i l l e, ~ a v r i l-s e p t e m b r e ~ 1984, ~ p . ~} 707$.

70. J. Hoed, «De l'information des quotidiens belges de langue française en matière de politique étrangère ", in Res Publica (VI-3), Bruxelles, 1964, p. 271.

71. AMAEF-A, dossier 21, sous-dossier Livres-abonnements, de de Vaucelles, chargé d'affaires ad interim, à Bidault, 30/8/1946. Chiffres confirmés par M. Stijns "La presse quotidienne d'information en Belgique et ses problèmes actuels ", in Études de presse (vol. III, n 2), Paris, Institut français de presse, 15 octobre 1951, p. 213. 